Федина А.А., Мкртчян A.C., Шевченко А.И., Шаталов Н.A.

Введение:

Результаты исследования:

Обсуждение

и заключение: Таким образом, в работе проведен анализ линеаризованной системы уравнений динамики атмосфреры. В эту систему входят уравнения движения в трех проекциях на оси координат, уравнение неразрывности и уравнение теплопроводности. Показано, что равенство нулю вертикальной составпяющей скорости является следствием стационарности возмущения температуры. Как правило, этот факт просто постулируется и входит в определение геострофического состояния атмоссеры.

Ключевые слова:

Северо-Кавказский федеральный университет

Россия, г. Ставрополь,

zakinyan@mail.ru

\section{ПОЛЯ ВОЗМУЩЕНИЯ ДАВЛЕНИЯ, ПЛОТНОСТИ И СКОРОСТИ ПРИ ГЕОСТРОФИЧЕСКОМ СОСТОЯНИИ АТМОСФЕРЫ}

Под общей (глобальной) циркуляцией атмоссеры (ОЦА) понимают совокупность воздушных течений (ветров) такой горизонтальной протяженности (масштаба), которая сравнима с размерами материков и океанов. К ОЦА относятся такие системы воздушных потоков, как западный перенос в умеренных широтах обоих полушарий, пассатные ветры субтропиков, муссоны, струйные течения, системы движения в планетарных волнах, циклонах и антициклонах. Циркуляция атмоссеры определяется рядом факторов, среди которых наиболее важными являются лучистая энергия Солнца, неравномерно распределенная на земном шаре и сила Кориолиса.

новным стационарным состоянием. При отом нам остается неизвесным поле возмущение давления. Поэтому в этой ситуации поступают следующим образом: по фактическому полю давления определяют скорость геострофического ветра. Получается, что геострофическое состояние является неопределенным. В данной работе предпагается решение для полей возмущения давпения и плотности, соответствующие геострофическому (стационарному) состоянию атмоссреры.

для возмущений давления и плотности получаем:

$p^{\prime}=-2 \omega_{0 z} \rho_{\mathrm{e}} \frac{c}{\sqrt{k_{1}^{2}+k_{2}^{2}}}\left(k_{1} x+k_{2} y\right), \quad \rho^{\prime}=\frac{2 \omega_{0 z} c}{g \sqrt{k_{1}^{2}+k_{2}^{2}}} \rho_{\mathrm{e}}\left[k_{2} \frac{\omega_{0 y}}{\omega_{0 z}}-\alpha\left(\gamma_{\mathrm{A}}-\gamma\right)\left(k_{1} x+k_{2} y\right)\right]$

Отсюда для проекций скорости геострофического ветра попучим выражения

$u_{\mathrm{g}}=-\frac{1}{2 \omega_{0 z} \rho_{\mathrm{e}}} \frac{\partial p^{\prime}}{\partial y}=\frac{k_{2}}{\sqrt{k_{1}^{2}+k_{2}^{2}}} c, \quad v_{\mathrm{g}}=\frac{1}{2 \omega_{0 z} \rho_{\mathrm{e}}} \frac{\partial p^{\prime}}{\partial x}=-\frac{k_{1}}{\sqrt{k_{1}^{2}+k_{2}^{2}}} c$ геострофическое состояние атмосферы, уравнение статики атмосферы геострофический ветер, сила Кориолиса, параметр Кориолиса, завихренность, функция тока 
Fedina A.A., North-Caucasian Federal University

Mkrtchyan A.S., Stavropol, Russia,

Shevchenko A.I., Shatalov N.A. zakinyan@mail.ru

\section{Fields of perturbation of pressure, density and speeds at a geostrophic state of atmosphere}

Introduction:

The general (global) atmospheric circulation (OCA) is understood as the totality of air currents (winds), which is the horizontal extent (scale), which allows you to get the size of the material and the oceans. Airflow systems such as westerly transport in temperate latitudes of both hemispheres, trade winds of subtropics, monsoons, string currents, a system of motion in planetary waves, cyclones and anticyclones. Atmospheric circulation is determined by a number of factors that are unevenly distributed across the globe and the Coriolis force.

Materials and methods:

As is known, the geostrophic state of the atmosphere is its basic stationary state. In this case, the pressure perturbation field remains unknown to us. Therefore, in this situation, proceed as follows: the actual pressure field determines the speed of the geostrophic wind. It turns out that the geostrophic state is uncertain. In this paper, a solution is proposed for pressure and density perturbation fields corresponding to the geostrophic (stationary) state of the atmosphere.

Results

of the research:

for pressure and density perturbations we get:

$p^{\prime}=-2 \omega_{0 z} \rho_{\mathrm{e}} \frac{c}{\sqrt{k_{1}^{2}+k_{2}^{2}}}\left(k_{1} x+k_{2} y\right), \quad \rho^{\prime}=\frac{2 \omega_{0 z} c}{g \sqrt{k_{1}^{2}+k_{2}^{2}}} \rho_{\mathrm{e}}\left[k_{2} \frac{\omega_{0 y}}{\omega_{0 z}}-\alpha\left(\gamma_{\mathrm{A}}-\gamma\right)\left(k_{1} x+k_{2} y\right)\right]$

From here for the projections of the speed of the geostrophic wind we obtain the expression

$u_{\mathrm{g}}=-\frac{1}{2 \omega_{0 z} \rho_{\mathrm{e}}} \frac{\partial p^{\prime}}{\partial y}=\frac{k_{2}}{\sqrt{k_{1}^{2}+k_{2}^{2}}} c, \quad v_{\mathrm{g}}=\frac{1}{2 \omega_{0 z} \rho_{\mathrm{e}}} \frac{\partial p^{\prime}}{\partial x}=-\frac{k_{1}}{\sqrt{k_{1}^{2}+k_{2}^{2}}} c$

Discussion

and conclusion:

Thus, the analysis of the linearized system of equations of atmospheric dynamics is carried out. This system includes the equations of motion in three projections on the coordinate axes, the continuity equation and the heat equation. It is shown that the zero vertical component of the velocity is a consequence of the stationarity of the temperature perturbation. As a rule, this fact is simply postulated and is included in the definition of the geostrophic state of the atmosphere.

Keywords: geostrophic state of the atmosphere, equation of atmospheric static, geostrophic wind, Coriolis force, Coriolis parameter, vorticity, flow function.

\section{Введение}

Под общей (глобальной) циркуляцией атмосферы (ОЦА) понимают совокупность воздушных течений (ветров) такой горизонтальной протяженности (масштаба), которая сравнима с размерами материков и океанов. К ОЦА относятся такие системы воздушных потоков, как западный перенос в умеренных широтах обоих полушарий, пассатные ветры субтропиков, муссоны, струйные течения, системы движения в планетарных волнах, циклонах и антициклонах [3]. 
Циркуляция атмосферы определяется рядом факторов, среди которых наиболее важными являются лучистая энергия Солнца, неравномерно распределенная на земном шаре и сила Кориолиса [2].

Представления об основных процессах ОЦА были заложены в работах В. Бьеркнеса и Россби еще в 1930-1940-х гг. [1].

Одним из основных элементов структуры ОЦА является западный ветер (западный перенос).

Как известно, крупномасштабные движения во вращающейся атмосфере являются квазистатическими и квазигеострофическими. Составляющие скорости горизонтального ветра легко определяются из геострофического соотношения, которые получаются в результате приравнивания градиента давления и силы Кориолиса [1].

\section{Материалы и методы исследования}

Как известно, геострофическое состояние атмосферы является ее основным стационарным состоянием. При этом нам остается неизвестным поле возмущение давления. Поэтому в этой ситуации поступают следующим образом: по фактическому полю давления определяют скорость геострофического ветра. Получается, что геострофическое состояние является неопределенным.

В данной работе предлагается решение для полей возмущения давления и плотности, соответствующие геострофическому (стационарному) состоянию атмосферы.

В локальной системе координат $(x, y, z)$ состояние атмосферы описывается системой уравнений

$$
\begin{aligned}
& \frac{\partial u}{\partial t}+u \frac{\partial u}{\partial x}+v \frac{\partial u}{\partial y}+w \frac{\partial u}{\partial z}=-\frac{1}{\rho}\left(\frac{\partial p}{\partial x}\right)+2 \omega_{0 z} v-2 \omega_{0 y} w \\
& \frac{\partial v}{\partial t}+u \frac{\partial v}{\partial x}+v \frac{\partial v}{\partial y}+w \frac{\partial v}{\partial z}=-\frac{1}{\rho}\left(\frac{\partial p}{\partial y}\right)-2 \omega_{0 z} u \\
& \frac{\partial w}{\partial t}+u \frac{\partial w}{\partial x}+v \frac{\partial w}{\partial y}+w \frac{\partial w}{\partial z}=-\frac{1}{\rho}\left(\frac{\partial p}{\partial z}\right)-g+2 \omega_{0 y} u, \\
& \frac{\partial T}{\partial t}+(\mathbf{v}, \nabla) T=0, \\
& \frac{\partial \rho}{\partial t}+u \frac{\partial \rho}{\partial x}+v \frac{\partial \rho}{\partial y}+w \frac{\partial \rho}{\partial z}+\rho\left(\frac{\partial u}{\partial x}+\frac{\partial v}{\partial y}+\frac{\partial w}{\partial z}\right)=0 .
\end{aligned}
$$

Здесь $T$ - температура воздуха возмущенной атмосферы, соответственно; 
$(u, v, w)-$ проекции скорости движения воздуха, соответственно, на оси $(x, y, z)$.

$\rho-\quad$ плотность воздуха;

$p-\quad$ давление воздуха;

$\left(\omega_{0 y}, \omega_{0 z}\right)-$ проекции вектора угловой скорости вращения Земли, соответственно, на оси $(y, z)$.

Напомним, что ось $x$ направлена по касательной к параллели в направлении вращения Земли, ось $y$ - по касательной к меридиану в направлении от экватора к северному полюсу; ось $z$ - перпендикулярна поверхности Земли. Поэтому $\omega_{0 x}=0$. Далее, $g-$ ускорение свободного падения.

Кинематическую вязкость воздуха и коэффициент температуропроводности воздуха считаем равными нулю.

Представим параметры атмосферы в возмущенном состоянии в виде:

$$
p=\bar{p}(z)+p^{\prime}(x, y, z, t), T=T_{\mathrm{e}}(z)+\theta(x, y, z, t), T_{\mathrm{e}}=T_{\mathrm{e} 0}-\gamma \cdot z, \rho=\rho_{\mathrm{e}}+\rho^{\prime}
$$

т.е. в виде суммы значения параметра в состояния статики атмосферы и возмущенного значения.

Уравнение статики атмосферы выражается формулами:

$$
\frac{\partial \bar{p}}{\partial x}=0, \frac{\partial \bar{p}}{\partial y}=0,-\frac{1}{\rho_{\mathrm{e}}}\left(\frac{\partial \bar{p}}{\partial z}\right)-g=0 .
$$

В состоянии статики плотность воздуха изменяется с высотой по закону:

$$
\rho_{\mathrm{e}}(z)=\rho_{\mathrm{e} 0} e^{-\alpha\left(\gamma_{\mathrm{A}}-\gamma\right) z}
$$

$$
\begin{array}{ll}
- & \text { распределение плотности воздуха с высотой в состоянии } \\
& \text { статики атмосферы; } \\
& \text { так называемый, градиент автоконвекции, он совпадает с } \\
& \text { градиентом температуры однородной по плотности атмос- } \\
\text { феры; } & \\
& T_{\mathrm{e}}(z)=T_{\mathrm{e} 0}-\gamma z \\
& \text { - температура воздуха в состоянии статики атмосферы; } \\
& \text { вертикальный градиент температуры. }
\end{array}
$$

Тогда система уравнений запишется в виде: 


$$
\begin{aligned}
& \frac{\partial u}{\partial t}+u \frac{\partial u}{\partial x}+v \frac{\partial u}{\partial y}+w \frac{\partial u}{\partial z}=-\frac{1}{\rho_{\mathrm{e}}}\left(\frac{\partial p^{\prime}}{\partial x}\right)+2 \omega_{0 z} v-2 \omega_{0 y} w, \\
& \frac{\partial v}{\partial t}+u \frac{\partial v}{\partial x}+v \frac{\partial v}{\partial y}+w \frac{\partial v}{\partial z}=-\frac{1}{\rho_{\mathrm{e}}}\left(\frac{\partial p^{\prime}}{\partial y}\right)-2 \omega_{0 z} u, \\
& \frac{\partial w}{\partial t}+u \frac{\partial w}{\partial x}+v \frac{\partial w}{\partial y}+w \frac{\partial w}{\partial z}=-\frac{1}{\rho_{\mathrm{e}}}\left(\frac{\partial p^{\prime}}{\partial z}\right)-\frac{\rho^{\prime}}{\rho_{\mathrm{e}}} g+2 \omega_{0 y} u, \\
& \frac{\partial \theta}{\partial t}+u \frac{\partial \theta}{\partial x}+v \frac{\partial \theta}{\partial y}+w \frac{\partial \theta}{\partial z}=\gamma \cdot w
\end{aligned}
$$

$$
\frac{\partial \rho^{\prime}}{\partial t}+u \frac{\partial \rho^{\prime}}{\partial x}+v \frac{\partial \rho^{\prime}}{\partial y}+w \frac{\partial \rho^{\prime}}{\partial z}+w \frac{\partial \rho_{\mathrm{e}}}{\partial z}+\left(\frac{\partial u}{\partial x}+\frac{\partial v}{\partial y}+\frac{\partial w}{\partial z}\right)=0 .
$$

Линеаризуем систему уравнений (10) - (14):

$$
\begin{aligned}
& \frac{\partial u}{\partial t}=-\frac{1}{\rho_{\mathrm{e}}} \frac{\partial p^{\prime}}{\partial x}+2 \gamma \omega_{0 z}-2 w \omega_{0 y}, \\
& \frac{\partial v}{\partial t}=-\frac{1}{\rho_{\mathrm{e}}} \frac{\partial p^{\prime}}{\partial y}-2 \omega_{0 z} u, \\
& \frac{\partial w}{\partial t}=-\frac{1}{\rho_{\mathrm{e}}} \frac{\partial p^{\prime}}{\partial z}-\frac{g}{\rho_{\mathrm{e}}} \rho^{\prime}+2 \omega_{0 y} u, \\
& \frac{1}{\rho_{\mathrm{e}}} \frac{\partial \rho^{\prime}}{\partial t}+\frac{\partial u}{\partial x}+\frac{\partial v}{\partial y}+\frac{\partial w}{\partial z}=\alpha\left(\gamma_{\mathrm{A}}-\gamma\right) w .
\end{aligned}
$$

$$
\frac{\partial \theta}{\partial t}=\gamma \cdot w
$$

Из уравнения (19) следует, что источником генерации вертикальной скорости является нестационарное возмущение температуры.

В стационарном состоянии

$$
\begin{aligned}
& -\frac{1}{\rho_{\mathrm{e}}} \frac{\partial p^{\prime}}{\partial x}+2 w \omega_{0 z}-2 w \omega_{0 y}=0, \\
& -\frac{1}{\rho_{\mathrm{e}}} \frac{\partial p^{\prime}}{\partial y}-2 \omega_{0 z} u=0, \\
& -\frac{1}{\rho_{\mathrm{e}}} \frac{\partial p^{\prime}}{\partial z}-\frac{g}{\rho_{\mathrm{e}}} \rho^{\prime}+2 \omega_{0 y} u=0,
\end{aligned}
$$




$$
\begin{aligned}
& \frac{\partial u}{\partial x}+\frac{\partial v}{\partial y}+\frac{\partial w}{\partial z}=\alpha\left(\gamma_{A}-\gamma\right) w \\
& w=0
\end{aligned}
$$

Обычно, равенство нулю вертикальной составляющей скорости является определением геострофического состояния. Но, как видно из приведенного анализа, равенство нулю вертикальной составляющей скорости является следствием стационарности состояния системы.

Получаем систему уравнений, описывающих геострофическое состояние атмосферы:

$$
\begin{aligned}
& -\frac{1}{\rho_{\mathrm{e}}} \frac{\partial p^{\prime}}{\partial x}+2 \omega_{0 z^{v}}=0, \\
& -\frac{1}{\rho_{\mathrm{e}}} \frac{\partial p^{\prime}}{\partial y}-2 \omega_{0 z} u=0, \\
& -\frac{1}{\rho_{\mathrm{e}}} \frac{\partial p^{\prime}}{\partial z}-\frac{g}{\rho_{\mathrm{e}}} \rho^{\prime}+2 \omega_{0 y} u=0, \\
& \frac{\partial u}{\partial x}+\frac{\partial v}{\partial y}=0 .
\end{aligned}
$$

Обычно при описании геострофического состояния атмосферы уравнение (22) заменяется уравнением статики атмосферы. Но на наш взгляд это не корректно. Нет оснований пренебрегать уравнением (22).

Взяв производную по переменной $x$ от (20) и производную по переменной $y$ от (21), и складывая уравнения, получим

$$
\begin{aligned}
\Omega_{z} & =\frac{1}{2 \omega_{0 z} \rho_{\mathrm{e}}}\left(\frac{\partial^{2} p^{\prime}}{\partial x^{2}}+\frac{\partial^{2} p^{\prime}}{\partial y^{2}}\right), \\
\text { Здесь } \quad & \Omega_{z}=\frac{\partial v}{\partial x}-\frac{\partial u}{\partial y} \\
& - \text { вертикальная завихренность }
\end{aligned}
$$

Из формулы (24) следует, что поле завихренности коррелирует с полем возмушения давления. Однако, наличие завихренности не означает, поле возмущения давления имеет круговой характер. По физическому смыслу геострофического состояния - это состояние с равным нулю ускорением. Поэтому движение может быть только прямолинейным и равномерным. 
Из (23) следует, что можно ввести функцию тока:

$$
u=\frac{\partial \psi}{\partial y}, \quad v=-\frac{\partial \psi}{\partial x} .
$$

Отсюда $\Omega_{z}=-\left(\frac{\partial^{2} \psi}{\partial x^{2}}+\frac{\partial^{2} \psi}{\partial y^{2}}\right)$.

Подставляя (27) в (24), получим

$$
-\left(\frac{\partial^{2}}{\partial x^{2}}+\frac{\partial^{2}}{\partial y^{2}}\right) \psi=\frac{1}{2 \omega_{0 z} \rho_{\mathrm{e}}}\left(\frac{\partial^{2}}{\partial x^{2}}+\frac{\partial^{2}}{\partial y^{2}}\right) p^{\prime},-\psi=\frac{1}{2 \omega_{0 z} \rho_{\mathrm{e}}} p^{\prime}
$$

Из (22) получим

$$
\rho^{\prime}=-\frac{1}{g}\left(\frac{\partial}{\partial z}+\frac{\omega_{0 y}}{\omega_{0 z}} \frac{\partial}{\partial y}\right) p^{\prime} \text {. }
$$

Умножим (20) на $v$, а (21) на $u$ и вычтем

$$
\frac{1}{\rho_{\mathrm{e}}}\left(v \frac{\partial}{\partial x}-u \frac{\partial}{\partial y}\right) p^{\prime}-2 \omega_{0 z}\left(u^{2}+v^{2}\right)=0 \text {. }
$$

Пусть $u^{2}+v^{2}=c^{2}=$ const .

$$
\left(\frac{\partial \psi}{\partial x}\right)^{2}+\left(\frac{\partial \psi}{\partial y}\right)^{2}=c^{2}
$$

Принятое допущение равносильно постоянству кинетической энергии движения воздуха, что является очевидным в стационарном состоянии атмосферы.

\section{Результаты исследования и их обсуждение}

Найдем решение уравнения (32) в виде:

$$
\psi=\frac{c}{\sqrt{k_{1}^{2}+k_{2}^{2}}}\left(k_{1} x+k_{2} y\right) .
$$

Отсюда следует, что линии тока в геострофическом состоянии атмосферы представляют собой прямые линии, направленные под углом к оси $x$, определяемым равенством: $\operatorname{tg} \alpha=k_{2} / k_{2}$.

Для возмущений давления и плотности соответственно получим

$$
\begin{aligned}
& p^{\prime}=-2 \omega_{0 z} \rho_{\mathrm{e}} \frac{c}{\sqrt{k_{1}^{2}+k_{2}^{2}}}\left(k_{1} x+k_{2} y\right), \\
& \rho^{\prime}=\frac{2 \omega_{0 z} c}{g \sqrt{k_{1}^{2}+k_{2}^{2}}} \rho_{\mathrm{e}}\left[k_{2} \frac{\omega_{0 y}}{\omega_{0 z}}-\alpha\left(\gamma_{\mathrm{A}}-\gamma\right)\left(k_{1} x+k_{2} y\right)\right] .
\end{aligned}
$$


Отсюда для проекций скорости геострофического ветра получим выражения

$$
u_{\mathrm{g}}=-\frac{1}{2 \omega_{0 z} \rho_{\mathrm{e}}} \frac{\partial p^{\prime}}{\partial y}=\frac{k_{2}}{\sqrt{k_{1}^{2}+k_{2}^{2}}} c, v_{\mathrm{g}}=\frac{1}{2 \omega_{0 z} \rho_{\mathrm{e}}} \frac{\partial p^{\prime}}{\partial x}=-\frac{k_{1}}{\sqrt{k_{1}^{2}+k_{2}^{2}}} c .
$$

Таким образом, в геострофическом состоянии атмосферы геострофический ветер имеет постоянное значение и направлен под углом $\operatorname{tg} \alpha=k_{2} / k_{2}$ к оси $x$. В зависимости от значений $k_{1}$ и $k_{2}$ направление геострофического ветра может быть произвольным. В частности, если $k_{1}=0$, и $k_{2}>0$ то мы имеем зональный поток в направлении с запада на восток. Если же $k_{1}=0$, а $k_{2}<0$, то мы имеем поток в обратном направлении с востока на запад.

Аналогично, если $k_{2}=0$, а $k_{2}<0$, то мы имеем меридиональный поток в направлении с юга на север. Если же $k_{2}=0$, a $k_{2}>0$, то мы имеем обратный поток с севера на юг.

В общем случае, если знаки $k_{1}$ и $k_{2}$ одинаковые, то мы имеем поток в направлении с северо-запада на юго-восток, если же знаки противоположны, то направление потока с юго-запада на северо-восток.

\section{Выводы}

Таким образом, в работе проведен анализ линеаризованной системы уравнений динамики атмосферы. В эту систему входят уравнения движения в трех проекциях на оси координат, уравнение неразрывности и уравнение теплопроводности. Показано, что равенство нулю вертикальной составляющей скорости является следствием стационарности возмущения температуры. Как правило, этот факт просто постулируется и входит в определение геострофического состояния атмосферы.

В систему уравнений, описывающих геострофическое состояние атмосферы, входит также вертикальная проекция уравнения движения. Данным уравнением, как правило, пренебрегают и заменяют ее уравнение статики атмосферы, что, на наш взгляд, является не правомерным.

Следует заметить, что во вращающейся атмосфере, вопреки установившимся представлениям, геострофического состояния быть не может. Это следует из того, что согласно первому закону Ньютона при равенстве нулю ускорения тело может двигаться только прямолинейно и равномерно. Поэтому о геострофическом движении можно говорить только локально в мальх масштабах.

Работа выполнена под научным руководством профессора Р.Г. Закиняна. 


\section{Библиографический список}

1. Гилл А. Динамика атмосфреры и океана. М.: Мир, 1986. Т. 1, 399 c.; T. 2, 416 c.

2. Holton J.R. An Introduction to Dynamic Meteorology. Forth edition. Elsevier, 2004, p. 540.

3. Матвеев Л.Т. Теория общей циркуляции атмосфреры и климата Земли. Л.: Гидрометеоиздат, 1991, 295 с.

4. Zakinyan R.G., Zakinyan A.R., Ryzhkov R. On the Isobaric Surface Shape in the Geostrophic State of the Atmosphere // The 1st International Electronic Conference on Atmospheric Sciences (ECAS 2016), 16-31 July 2016; Sciforum Electronic Conference Series. Vol. 1, 2016.

5. Zakinyan R.G., Zakinyan A.R., Ryzhkov R. Phases of the Isobaric Surface Shapes in the Geostrophic State of the Atmosphere and Connection to the Polar Vortices // Atmosphere 2016, 7, 126; doi: $10.3390 /$ atmos 7100126 .

6. Шакина Н.П. Лекции по динамической метеорологии. М.: Триада ЛТД, 2013. $160 \mathrm{c}$

\section{References}

1. Gill A. Dynamics of the atmosphere and the ocean. M.: Mir, 1986, T. 1, 399 p.; T. 2, $416 \mathrm{~s}$.

2. Holton J.R. An Introduction to Dynamic Meteorology. Forth edition. Elsevier, 2004, p. 540.

3. Matveyev L.T. The theory of the general circulation of the atmosphere and climate of the Earth. L.: Hydrometeoizdat, 1991, 295 p.

4. Zakinyan R.G., Zakinyan A.R., Ryzhkov R. On the Isobaric Surface Shape in the Geostrophic State of the Atmosphere // The 1st International Electronic Conference on Atmospheric Sciences (ECAS 2016), 16-31 July 2016; Sciforum Electronic Conference Series, Vol. 1, 2016.

5. Zakinyan R.G., Zakinyan A.R., Ryzhkov R. Phases of the Isobaric Surface Shapes of the Atmosphere and the Polar Vortices // Atmosphere 2016. 7. 126. doi: 10.3390 / atmos 7100126

6. Shakina N.P. Lectures on dynamic meteorology. M.: TRIADA LTD, 2013. $160 \mathrm{p}$.

Рукопись поступила в редакциго 13.12.19, принята к публикации 01.03.19.

\section{6 aвторах}

Федина А.А., аспирант кафедры общей и теоретической физики Северо-Кавказского федерального университета. Тел. 8-918-757-76-55.

E-mail: alexmendas@gmail.com.

Мкртчян А.C., аспирант кафедры общей и теоретической физики Северо-Кавказского федерального университета. Тел. 8-918-779-60-50. E-mail: Mkrtchyan-asmik@mail.ru. 
Шевченко А.И, доцент кафедры общей и теоретической физики Северо-Кавказского фредерального университета. Тел. 8-905-499-12-01.

E-mail: shev-ai@mail.ru.

Шаталов Н.A., аспирант кафедры общей и теоретической ффизики СевероКавказского федерального университета.

\section{About the authors}

Fedina A.A., Post-Graduate Student, Department of General and Theoretical Physics, North-Caucasian Federal University. Phone: 8-918-757-76-55. Email: alexmendas@gmail.com.

Mkrtchyan A.S., Post-Graduate Student Department of General and Theoretical Physics, North-Caucasian Federal University Phone: 8-918-779-60-50. E-mail: Mkrtchyan-asmik@mail.ru.

Shevchenko A.I., Associate Professor of the Department of General and Theoretical Physics of the North-Caucasian Federal University, Phone: 8-905-499-12-01, E-mail: shev-ai@mail.ru.

Shatalov N.A., Post-Graduate Student, Department of General and Theoretical Physics, North-Caucasian Federal University

$\prod_{b}$

Издательство Северо-Кавказского

федерального университета

г. Ставрополь, ул. Пушкина, 1.

Корректор - М.И. Толмачев

Компьютерная верска - О.Г. Полевич.

Подписано в печать 29.03.2019 г. Выход в свет 09.04.2019 г Формат 70 × 108 1/16. Гарнитура Times New Roman. Бумага офсетная. Усл. печ. л. 15,5. Тираж 1000 экз. Цена 534 руб.

Отпечатано в Издательско-полиграфическом комплексе ФГАОУВО «Северо-Кавказскийфедеральныйуниверситет». 355029, г. Ставрополь, пр-т Кулакова, 2 